Неклассическая логика

Non-classical Logic

Л.Ю. ДЕвяткин

\title{
Неклассические модификации многозначных матриц классической логики. Часть II
}

\author{
Девяткин Леонид Юрьевич \\ Сектор логики, Институт философии РАН \\ Российская Федерация, 109240, г. Москва, ул. Гончарная, д. 12, стр. 1. \\ E-mail: leoniddevyatkin@gmail.com
}

Данная статья является второй в дилогии, посвященной многозначным матрицам классической пропозициональной логики как инструменту построения и анализа неклассических логик. В литературе существует множество пар трехзначных матриц, различающихся лишь классами выделенных значений. Но подавляющее большинство из них задает неклассическое отношение следования как при одном выделенном значении, так и при двух. Однако существуют матрицы неклассических логик, полученные из матриц классической логики сужением или расширением класса выделенных значений. Основная часть статьи посвящена двум классам матриц. Первый класс состоит из матриц, которые задавали бы классическое отношение следования при $D=\{1,2\}$, однако рассматриваются с $D=\{2\}$. Второй класс получен выбором $D=\{1,2\}$ в матрицах, порождающих классическое следование при $D=\{2\}$. Для изучаемых матриц доказывается максимальность (в сильном смысле) паранепротиворечивости или параполноты задаваемых ими логик, а также аналоги теоремы Гливенко или дуальной теоремы Гливенко. Матрицы в рассматриваемых классах образуют решетки по отношению функциональной вложимости. Отдельные матрицы, полученные из матриц классической логики модификацией множества выделенных значений, имеют эквивалентные формулировки в виде функциональных расширений матриц классической логики.

Ключевые слова: многозначные логики, логические матрицы, паранепротиворечивость, параполнота

(C) Девяткин Л.Ю. 


\section{1. Введение}

Хорошо известно, что на основе одной и той же алгебры можно построить матрицы, задающие разные логики. В этом случае различия между логиками определяются выбором классов выделенных значений. В Части I работы мы неоднократно сталкивались с примерами таких матриц. В частности, это $E_{3}$ и $J_{3}, B_{3}$ и $S_{3}, P^{1}$ и $I^{1}, K_{3}$ и $L P$.

Зачастую матрицы, различающиеся лишь классами выделенных значений, бывают построены независимо, и их функциональную эквивалентность открывают позже. Так, матрица $J_{3}$ была впервые предложена в 1970 г. [15], однако первое известное автору указание на ее связь с $E_{3}$ относится к 1985 г. [14]. Матрица $S_{3}$ строилась К. Сегербергом в [45] как расширение матрицы С. Холдена, вне связи с $B_{3}$. Как указывают А.С. Карпенко и Н. Томова [29, §2.5], взаимовыразимость операций $P^{1}$ и $I^{1}$ была явным образом установлена только в 2000 г.

В то же время Г. Прист изначально строит $L P$, расширяя класс выделенных значений матрицы $K_{3}$ [38]. Эти две матрицы нередко рассматривают параллельно. Во-первых, они могут трактоваться как подматрицы четырехзначной матрицы Данна и Белнапа $B_{4}$ (см., например, [39], [50]). В этом случае промежуточное значение в $K_{3}$ трактуется как «ни истинно, ни ложно», а в $L P$ - как «истинно и ложно одновременно». Альтернативную трактовку дает Д. Рипли [44]. Он в обоих случаях интерпретирует промежуточное значение как «истинно и ложно одновременно», а различие между матрицами вытекает из критериев выбора выделенных значений. В случае $K_{3}$ предложению приписывается выделенное значение, если оно по меньшей мере истинно. В случае $L P$ - если оно не ложно.

В общих терминах влияние выбора класса выделенных значений на свойства логики, задаваемой матрицей, анализируется в книге Р.Л. Эпштейна [17, p. 285-287]. Автор обращает внимание на то, что условия стандартности Россера-Тюркетта могут нарушаться двумя способами: операция многозначной матрицы может принимать невыделенное значение, когда, согласно соответствующему условию стандартности, должна была принять выделенное, или она может принимать выделенное значение на значениях аргументов, для которых 
выполнение условия стандартности требовало бы невыделенного значения. Следуя этой линии рассуждения, А. Бруннер и В. Карниэлли пишут [5]: «Интуиционистские логики являются "ложными по умолчанию" (в том смысле, что предложение и его отрицание могут оба приниматься как ложные), в то время как паранепротиворечивые логики являются “истинными по умолчанию” (в том смысле, что предложение и его отрицание могут оба приниматься как истинные)» (пер. автора).

Все трехзначные матрицы неклассических логик, которые мы рассматривали до этого, задают неклассическое отношение логического следования вне зависимости от выбора класса выделенных значений. Все они содержат операции, которые делают их «ложными по умолчанию» или «истинными по умолчанию» в смысле Бруннера и Карниэлли при $D=\{2\}$ и $D=\{1,2\}$. В некоторых случаях это инволюция, а в остальных это пара отрицаний из $P^{1}$ и $I^{1}$. Однако в литературе есть и отдельные примеры матриц, которые получены из матриц классической логики одним лишь изменением класса выделенных значений, без изменения операций. Матрица, в которой все операции отвечают условиям стандартности при $D=\{2\}$ приобретает «истинные по умолчанию» операции, когда происходит «переоценка» невыделенного промежуточного значения. В свою очередь, матрица, в которой все операции отвечают условиям стандартности при $D=\{1,2\}$ приобретает «ложные по умолчанию» операции, когда происходит «недооценка» выделенного промежуточного значения. Настоящая работа посвящена систематическому изучению матриц такого типа.

Материал организован следующим образом. В оставшейся части введения я рассматриваю примеры интересующих нас матриц, известные в литературе. Это последовательность матриц Гёделя, «ненормальная характеристическая матрица классической логики» А. Чёрча, а также матрица логики рационального агента Е.А. Кубышкиной и Д.В. Зайцева. После этого я определяю два дуальных класса трехзначных матриц, которые получены из матриц классической логики «недоценкой»и «переоценкой» промежуточных значений, эти классы обозначаются как $T L_{1}$ и $T L_{2}$ соответственно. Далее, 
исследуются свойства логик, задаваемых матрицами из этих классов. Показано, что матрицы из класса $T L_{2}$ задают логики, максимально паранепротиворечивые в сильном смысле, согласно определению О. Ариэли и соавторов. В силу дуальности, матрицы из класса $T L_{1}$ задают логики, максимально параполные в сильном смысле. Доказывается ряд утверждений, выступающих аналогами теоремы Гливенко и дуальной теоремы Гливенко. Потом я перехожу к рассмотрению функциональных свойств исследуемых матриц. Элементы классов $T L_{1}$ и $T L_{2}$ образуют решетки по отношению функциональной вложимости. А их подклассы $C$-расширяющих матриц - собственные подрешетки соответствующих решеток. Кроме того, я демонстрирую, что некоторые из матриц, полученных «недооценкой» или «переоценкой» промежуточных значений могут быть также представлены как функциональные расширения матриц классической логики. Заключение посвящено проблемам обобщения результатов, изложенных в Части I и Части II работы.

Предполагается знакомство читателя с предыдущей частью статьи, в ней можно найти все недостающие определения и ссылки, необходимые для понимания настоящего текста.

Теперь перейдем к рассмотрению примеров матриц с «недоценкой» и «переоценкой» промежуточных значений. Исторически первый пример дают нам многозначные матрицы Гёделя. Операции матрицы $G_{n}=\langle\{0,1, \ldots, n-1\}, \wedge, \vee, \Rightarrow, \neg,\{n-1\}\rangle$ отвечают следующим условиям [20]:

$$
\begin{gathered}
a \wedge b=\min (a, b) ; a \vee b=\max (a, b) ; \\
a \Rightarrow b=\left\{\begin{array}{l}
n-1, \text { если } a \leq b ; \\
b, \text { если } a>b .
\end{array} \quad \neg a=\left\{\begin{array}{l}
0, \text { если } a \neq 0 ; \\
n-1, \text { если } a=0 .
\end{array}\right.\right.
\end{gathered}
$$

Для трехзначной матрицы $G_{3}=\langle\{0,1,2\}, \wedge, \vee, \Rightarrow, \neg,\{2\}\rangle$ получаем следующие таблицы:

\begin{tabular}{|l|lll|}
\hline$\wedge$ & 0 & 1 & 2 \\
\hline 0 & 0 & 0 & 0 \\
1 & 0 & 1 & 1 \\
2 & 0 & 1 & 2 \\
\hline
\end{tabular}

\begin{tabular}{|c|ccc|}
\hline$\vee$ & 0 & 1 & 2 \\
\hline 0 & 0 & 1 & 2 \\
1 & 1 & 1 & 2 \\
2 & 2 & 2 & 2 \\
\hline
\end{tabular}

\begin{tabular}{|c|ccc|}
\hline$\Rightarrow$ & 0 & 1 & 2 \\
\hline 0 & 2 & 2 & 2 \\
1 & 0 & 2 & 2 \\
2 & 0 & 1 & 2 \\
\hline
\end{tabular}

\begin{tabular}{|c|c|}
\hline & $\neg x$ \\
\hline 0 & 2 \\
1 & 0 \\
2 & 0 \\
\hline
\end{tabular}


Нетрудно увидеть, что при $D=\{1,2, \ldots, n-1\}$ (в трехзначном случае $-\{1,2\})$ операции $G_{n}$ отвечают условию стандартности Россера-Тюркетта ${ }^{1}$. Таким образом, матрицы Гёделя получены из матриц классической логики «недооценкой» промежуточных значений.

Бруннер и Карниэлли сопоставляют последовательности матриц Гёделя последовательность дуальных «анти-интуиционистских» матриц [5]. Она состоит из матриц вида $G_{n}^{*}=\langle\{0,1, \ldots, n-$ $\left.1\}, \wedge, \vee,-, \neg^{*},\{1, \ldots, n-1\}\right\rangle$, где операции отвечают следующим условиям:

$$
\begin{aligned}
& a \wedge b=\min (a, b) ; a \vee b=\max (a, b) ; \\
& a-b=\left\{\begin{array}{l}
0, \text { если } a \leq b ; \\
a, \text { если } a>b .
\end{array} \quad \neg^{*} a=\left\{\begin{array}{l}
0, \text { если } a=n-1 ; \\
n-1, \text { если } a \neq n-1 .
\end{array}\right.\right.
\end{aligned}
$$

Использование операции $x-y$, которую называют «псевдоразностью» или «исключением», в качестве дуала $x \Rightarrow y$ восходит к работе МакКинси и Тарского [36]. Такой подход является обычным в работах по данной теме (см., например, [42], [21], [56], [22], [57]). Однако отметим, вслед за Т. Фергюсоном [18], что не существует такой функции $\iota$ на $\{0,1, \ldots, n-1\}$, для которой $\iota(\iota(x) \Rightarrow \iota(y))=(x-y)$ или $\iota(\iota(x)-\iota(y))=(x \Rightarrow y)$, и в то же время для инволюции $\sim$ выполняются тождества: $\sim(\sim x \Rightarrow \sim y)=(y-x) ; \sim(\sim y-\sim x)=(x \Rightarrow y)$. Поэтому, принимая во внимание построения, касающиеся дуализации, из Части I данной работы ${ }^{2}$, в качестве дуала $x \Rightarrow y$ я буду рассматривать операцию $x \Leftarrow y=y-x$, как это делают А. Монтейро [37, Th. 2.6] и А.С. Карпенко [27]. Она отвечает следующему условию:

$$
a \Leftarrow b=\left\{\begin{array}{l}
0, \text { если } a \geq b ; \\
b, \text { если } a<b .
\end{array}\right.
$$

Для наглядности, рассмотрим трехзначную матрицу $G_{3}^{*}=$ $\left\langle\{0,1,2\}, \wedge, \vee, \leftarrow, \neg^{\diamond},\{1,2\}\right\rangle$. Таблицы для $\Leftarrow, \neg^{*}$ таковы:

\footnotetext{
${ }^{1}$ См. Часть I.

${ }^{2}$ См. также [34].
} 


\begin{tabular}{|c|ccc|}
\hline$\Leftarrow$ & 0 & 1 & 2 \\
\hline 0 & 0 & 1 & 2 \\
1 & 0 & 0 & 2 \\
2 & 0 & 0 & 0 \\
\hline
\end{tabular}

\begin{tabular}{|c|c|}
\hline & $\neg^{*} x$ \\
\hline 0 & 2 \\
1 & 2 \\
2 & 0 \\
\hline
\end{tabular}

Ясно, что при $D=\{n-1\}$ (в трехзначном случае $-\{2\})$ операции $G_{n}^{*}$ отвечают условию стандартности Россера-Тюркетта. То есть, матрицы вида $G_{n}^{*}$ получены из матриц классической логики в результате «переоценки» промежуточных значений.

В работе [7] А. Чёрч приводит еще одну матрицу интересующего нас типа. В цитируемой статье автор рассматривает матрицы, которые не являются «нормальными в смысле Карнапа», и в то же время являются характеристическим для классической пропозициональной логики. Матрица является «нормальной» в смысле Карнапа, если и только если она является «стандартной» в смысле Россера-Тюркетта. Матрица называется характеристической для некоторого исчисления, если и только если класс ее законов совпадает с классом теорем данного исчисления. В качестве одного из примеров Чёрч рассматривает матрицу, где $D=\{2\}$, а операции определяются такими таблицами:

\begin{tabular}{|c|ccc|}
\hline$\wedge^{\diamond}$ & 0 & 1 & 2 \\
\hline 0 & 0 & 0 & 0 \\
1 & 0 & 2 & 2 \\
2 & 0 & 2 & 2 \\
\hline
\end{tabular}

\begin{tabular}{|c|ccc|}
\hline$\vee^{\diamond}$ & 0 & 1 & 2 \\
\hline 0 & 0 & 2 & 2 \\
1 & 2 & 2 & 2 \\
2 & 2 & 2 & 2 \\
\hline
\end{tabular}

\begin{tabular}{|c|ccc|}
\hline$\supset^{\diamond}$ & 0 & 1 & 2 \\
\hline 0 & 2 & 2 & 2 \\
1 & 0 & 2 & 2 \\
2 & 0 & 2 & 2 \\
\hline
\end{tabular}

\begin{tabular}{|c|c|}
\hline & $\neg^{\diamond} x$ \\
\hline 0 & 2 \\
1 & 0 \\
2 & 0 \\
\hline
\end{tabular}

Такую же матрицу рассматривал Н. Решер как «слабый» вариант логики Лукасевича [43, p. 32-33]. А.С. Карпенко обратил внимание на то, что аналогичные операции выразимы и в матрице Бочвара $B_{3}$, образуя второй набор «внешних» операций [27, с. 53]. Таким образом, как указал Карпенко, логика Бочвара содержит не один фрагмент, изоморфный классическому исчислению высказываний, а два - заданный матрицей $B_{3}^{\square}$ с внешними операциями, определенными самим Бочваром, и $B_{3}^{\diamond}$ с операциями, отвечающими таблицам, изображенным выше. 
Как и в случае матриц Гёделя, матрице $B_{3}^{\diamond}$ можно сопоставить дуальную ей. В работе [12] рассматривается матрица с $D=\{1,2\}$ и следующими операциями:

\begin{tabular}{|c|ccc|}
\hline$\wedge^{\square}$ & 0 & 1 & 2 \\
\hline 0 & 0 & 0 & 0 \\
1 & 0 & 0 & 0 \\
2 & 0 & 0 & 2 \\
\hline
\end{tabular}

\begin{tabular}{|c|ccc|}
\hline$\vee^{\square}$ & 0 & 1 & 2 \\
\hline 0 & 0 & 0 & 2 \\
1 & 0 & 0 & 2 \\
2 & 2 & 2 & 2 \\
\hline
\end{tabular}

\begin{tabular}{|c|ccc|}
\hline$\supset^{\square}$ & 0 & 1 & 2 \\
\hline 0 & 2 & 2 & 2 \\
1 & 2 & 2 & 2 \\
2 & 0 & 0 & 2 \\
\hline
\end{tabular}

\begin{tabular}{|c|c|}
\hline & $\neg^{\square} x$ \\
\hline 0 & 2 \\
1 & 2 \\
2 & 0 \\
\hline
\end{tabular}

Нетрудно убедиться, что выполняются следующие тождества: $x \wedge^{\square} y=\sim\left(\sim x \vee^{\diamond} \sim y\right) ; x \wedge^{\diamond} y=\sim\left(\sim x \vee^{\square} \sim y\right) ; x \vee^{\square} y=\sim\left(\sim x \wedge^{\diamond} \sim y\right) ;$ $x \vee^{\diamond} y=\sim\left(\sim x \wedge^{\square} \sim y\right) ; \neg^{\square} x=\sim \neg^{\diamond} \sim x ; \neg^{\diamond} x=\sim \neg^{\square} \sim x$. Дуалом к $\supset^{\square}$, согласно нашей процедуре, окажется $x \subset^{\square} y=: \sim\left(\sim x \supset^{\square} \sim y\right)$. Но в то же время имеет место $x \supset^{\square} y=\neg^{\square}\left(\neg^{\square} x \subset^{\square} \neg^{\square} y\right) ; x \subset^{\square} y=$ $\neg^{\square}\left(\neg^{\square} x \supset^{\square} \neg^{\square} y\right)$.

Описанная матрица совпадает с фрагментом $B_{3}^{\square}$ трехзначной матрицы Бочвара, однако отличается от него классом выделенных значений. Заметим, что матрица трехзначной логики Сегерберга $S_{3}$ имеет тот же набор операций, что у Бочвара, но два выделенных значения. Поэтому матрицу $S_{3}^{\square}=\left\langle\{0,1,2\}, \wedge^{\square}, \vee^{\square}, \supset^{\square}, \neg^{\square},\{1,2\}\right\rangle$ можем трактовать как фрагмент матрицы Сегерберга.

Заключительный пример из литературы представляет наибольший интерес, т. к. в нем «недооценка» истинностных значений проявляется в наиболее явном виде. Д.В. Зайцев и Е.А. Кубышкина [31] строят четырехзначную логику, в которой истинностные значения имеют составную природу. Элементы множества $\{F, T\}$ интерпретируются как «онтологически ложно» и «онтологически истинно», а элементы множества $\{0,1\}$ как «не известно» и «известно». Множеством-носителем матрицы, которую строят авторы, оказывается произведение этих двух множеств: $\{F 0, F 1, T 0, T 1\}$. Адаптируя обозначения к терминологии текущей работы, будем далее писать $\{0,1,2,3\}$. Тогда интересующая нас матрица приобретает вид $L R A=\langle\{0,1,2,3\}, \wedge, \vee, \neg, \sim,\{3\}\rangle$. Таблицы для базовых операций таковы: 


\begin{tabular}{|l|llll|}
\hline$\wedge$ & 0 & 1 & 2 & 3 \\
\hline 0 & 0 & 0 & 0 & 0 \\
1 & 0 & 1 & 1 & 1 \\
2 & 0 & 1 & 2 & 2 \\
3 & 0 & 1 & 2 & 3 \\
\hline
\end{tabular}

\begin{tabular}{|c|cccc|}
\hline$\vee$ & 0 & 1 & 2 & 3 \\
\hline 0 & 0 & 1 & 2 & 3 \\
1 & 1 & 1 & 2 & 3 \\
2 & 2 & 2 & 2 & 3 \\
3 & 3 & 3 & 3 & 3 \\
\hline
\end{tabular}

\begin{tabular}{|c|c|}
\hline & $\neg x$ \\
\hline 0 & 2 \\
1 & 3 \\
2 & 0 \\
3 & 1 \\
\hline
\end{tabular}

\begin{tabular}{|c|c|}
\hline & $\sim x$ \\
\hline 0 & 1 \\
1 & 0 \\
2 & 3 \\
3 & 2 \\
\hline
\end{tabular}

Как отмечают Кубышкина и Зайцев, «если мы определяем отношение следования классическим образом (истинные посылки должны влечь истинные заключения), мы получаем классическую логику, где различие между известными и не известными истинами отсутствует» (пер. автора). То есть речь идет о том, что если мы полагаем $D=\{2,3\}$, то $L R A$ есть матрица классической логики. Многозначность возникает, когда происходит «недооценка» предложений, которые описывают положение дел, имеющее место в действительности, но не известное познающему субъекту, и их отказываются трактовать как истинные по эпистемическим соображениям. Чтобы нагляднее проиллюстрировать, связь $L R A$ с классической логикой, покажем, что она функционально эквивалентна матрице $L R A^{*}=\langle\{0,1,2,3\}, \wedge, \vee, \supset,-,\{3\}\rangle$, в которой базовые операции отвечают условиям стадартности Россера-Тюркетта при $D=\{2,3\}$. Импликация и отрицание отвечают таблицам ниже:

\begin{tabular}{|l|llll|}
\hline$\supset$ & 0 & 1 & 2 & 3 \\
\hline 0 & 2 & 2 & 2 & 2 \\
1 & 3 & 3 & 3 & 2 \\
2 & 0 & 0 & 3 & 2 \\
3 & 1 & 0 & 3 & 2 \\
\hline
\end{tabular}

\begin{tabular}{|c|c|}
\hline & $-x$ \\
\hline 0 & 3 \\
1 & 2 \\
2 & 1 \\
3 & 0 \\
\hline
\end{tabular}

Покажем функциональную эквивалентность $L R A$ и $L R A^{*}$ следующими тождествами: $-x=\sim \neg x ; x \supset y=\neg(x \wedge-y) ; \neg x=x \supset$ $(x \wedge-x) ; \sim x=-\neg x$.

Приведенные выше примеры служат мотивом для более систематического изучения матриц классической логики с «переоценкой» и «недооценкой» истинностных значений. В дальнейшем будем называть их $T L$-матрицами, от английского «true lies». В следующем разделе я начну с рассмотрения двух классов трехзначных $T L$-матриц: класса $T L_{1}$ матриц с одним выделенным значением и $T L_{2}$ с двумя, - 
а позже сделаю ряд обобщений для большего числа истинностных значений.

\section{2. ллассы матриц $T L_{1}$ и $T L_{2}$}

Построим класс трехзначных $T L_{1}$ матриц, в которых базовые операции отвечают условиям стандартности Россера-Тюркетта при $D=$ $\{1,2\}$, однако класс выделенных значений ограничен одним элементом. Он состоит из матриц, имеющих вид $M=\langle\{0,1,2\}, \wedge, \vee, \rightarrow$, $\dot{\neg},\{2\}\rangle$, в которых операции отвечают следующим таблицам:

\begin{tabular}{|c|ccc|}
\hline$\wedge$ & 0 & 1 & 2 \\
\hline 0 & 0 & 0 & 0 \\
1 & 0 & 1 или 2 & 1 или 2 \\
2 & 0 & 1 или 2 & 1 или 2 \\
\hline
\end{tabular}

\begin{tabular}{|c|ccc|}
\hline$\vee$ & 0 & 1 & 2 \\
\hline 0 & 0 & 1 или 2 & 1 или 2 \\
1 & 1 или 2 & 1 или 2 & 1 или 2 \\
2 & 1 или 2 & 1 или 2 & 1 или 2 \\
\hline
\end{tabular}

\begin{tabular}{|c|ccc|}
\hline$\rightarrow$ & 0 & 1 & 2 \\
\hline 0 & 1 или 2 & 1 или 2 & 1 или 2 \\
1 & 0 & 1 или 2 & 1 или 2 \\
2 & 0 & 1 или 2 & 1 или 2 \\
\hline
\end{tabular}

\begin{tabular}{|c|c|}
\hline & $\dot{\neg} x$ \\
\hline 0 & 1 или 2 \\
1 & 0 \\
2 & 0 \\
\hline
\end{tabular}

Класс $T L_{2}$ получаем с помощью процедуры дуализации, описанной в Части I. В матрицах этого класса операции отвечают условиям стандартности при одном выделенном значении, однако $D=\{1,2\}$. Элементы $T L_{2}$ имеют вид $M=\langle\{0,1,2\}, \wedge, \vee, \leftarrow, \ddot{ᄀ},\{1,2\}\rangle$, а их операции отвечают следующим таблицам:

\begin{tabular}{|c|ccc|}
\hline$\wedge$ & 0 & 1 & 2 \\
\hline 0 & 0 или 1 & 0 или 1 & 0 или 1 \\
1 & 0 или 1 & 0 или 1 & 0 или 1 \\
2 & 0 или 1 & 0 или 1 & 2 \\
\hline
\end{tabular}

\begin{tabular}{|c|ccc|}
\hline$\vee$ & 0 & 1 & 2 \\
\hline 0 & 0 или 1 & 0 или 1 & 2 \\
1 & 0 или 1 & 0 или 1 & 2 \\
2 & 2 & 2 & 2 \\
\hline
\end{tabular}

\begin{tabular}{|c|ccc|}
\hline$\leftarrow$ & 0 & 1 & 2 \\
\hline 0 & 0 или 1 & 0 или 1 & 2 \\
1 & 0 или 1 & 0 или 1 & 2 \\
2 & 0 или 1 & 0 или 1 & 0 или 1 \\
\hline
\end{tabular}

\begin{tabular}{|c|c|}
\hline & $\ddot{7} x$ \\
\hline 0 & 2 \\
1 & 2 \\
2 & 0 или 1 \\
\hline
\end{tabular}


Обычно, когда классы матриц задаются через условия, накладываемые на свойства базовых операций, требуют, чтобы эти операции были, помимо прочего, $C$-расширяющими (см., например, $[6$, $\S 5.3],[1],[19],[54]$, [51]). Однако я воздерживаюсь от этого требования в пользу более обобщенного подхода. Обратим внимание, что рассмотренная выше матрица $L R A$ не является $C$-расширяющей. В то же время необходимо отметить, что отказ от обсуждаемого ограничения ведет к определенным затруднениям. В классе $T L_{1}$ появляется «вырожденная» матрица, в которой каждая неэлементарная формула принимает только значения из $\{0,1\}$ - множества невыделенных значений. Аналогично, в $T L_{2}$ имеется элемент, область значений элементарных операций которого ограничена $\{1,2\}$, т. е. выделенными значениями. Обозначим эти матрицы как $B_{3}^{\star}$ и $S_{3}^{\star}$ соответственно. Ниже привожу по две операции для каждой матрицы, остальные определяются через них так же, как в классической логике.

\begin{tabular}{|c|ccc|}
\hline$\rightarrow_{B}$ & 0 & 1 & 2 \\
\hline 0 & 1 & 1 & 1 \\
1 & 0 & 1 & 1 \\
2 & 0 & 1 & 1 \\
\hline
\end{tabular}

\begin{tabular}{|c|c|}
\hline & $\neg_{B} x$ \\
\hline 0 & 1 \\
1 & 0 \\
2 & 0 \\
\hline
\end{tabular}

\begin{tabular}{|c|ccc|}
\hline$\leftarrow_{S}$ & 0 & 1 & 2 \\
\hline 0 & 1 & 1 & 2 \\
1 & 1 & 1 & 2 \\
2 & 1 & 1 & 1 \\
\hline
\end{tabular}

\begin{tabular}{|c|c|}
\hline & $\neg_{S} x$ \\
\hline 0 & 2 \\
1 & 2 \\
2 & 1 \\
\hline
\end{tabular}

Едва ли можно считать $\neg B x$ и $\neg S x$ полноценными отрицаниями. По своим свойствам эти операторы ближе к $\perp$ и $\top$ (см. [23, § 1.3.], а также [6, p. 11-12]). При этом в классах $T L_{1}$ и $T L_{2}$ значительное количество матриц не содержит более удачных кандидатов на роль отрицания. Однако, по мнению автора, существует и достаточно веский аргумент, оправдывающий включение соответствующих матриц в изучаемый класс. Чтобы изложить его, потребуется ввести дополнительные понятия.

До этого мы пользовались определением следования в терминах логических матриц. Теперь расширим это понятие. Отношением следования по Тарскому для пропозиционального языка $\mathcal{L}$ называем бинарное отношение $\vdash$ между $X \subseteq \operatorname{For}(\mathcal{L})$ и $\alpha \in \operatorname{For}(\mathcal{L})$, отвечающее трем условиям:

- Если $\alpha \in X$, то $X \vdash \alpha$ (рефлексивность); 
- Если $X \vdash \alpha$ и $X \subseteq X^{\prime}$, то $X^{\prime} \vdash \alpha$ (монотонность);

- Если $X \vdash \alpha$ и $X^{\prime} \vdash \alpha$, то $X, X^{\prime} \vdash \alpha$ (транзитивность).

Называем $\vdash$ структурным, если для каждого эндоморфизма $\theta$ в $\mathcal{L}$, каждого множества формул $X$ и каждой формулы $\alpha$ имеет место: если $X \vdash \alpha$, то $\theta(X) \vdash \theta(\alpha)$. Называем $\vdash$ нетривиальным, если найдутся непустое множество формул $X$ и формула $\alpha$, такие что $X \nvdash \alpha$. Называем $\vdash$ финитарным, если для каждого множества формул $X$ и каждой формулы $\alpha$, таких что $X \vdash \alpha$, найдется конечное множество $X^{\prime}$, для которого выполняется: $X^{\prime} \subseteq X$ и $X^{\prime} \vdash \alpha$. Если $\vdash$ структурное, нетривиальное и финитарное следование по Тарскому для пропозиционального языка $\mathcal{L}$, пара $\langle\mathcal{L}, \vdash\rangle$ назовем пропозичиональной логикой.

Имеет место следующий факт. Пусть $X \vdash \alpha$, е.т.е. $\langle X, \alpha\rangle \in$ $C n(M)$ для некоторой конечнозначной матрицы $M$, в которой $D$ есть непустое собственное подмножество множества-носителя. Тогда $\langle\mathcal{L}, \vdash\rangle$ есть пропозициональная логика. Структурность $\vdash$ вытекает из [58, § 3.1.3], финитарность - из [48], нетривиальность - из ограничения, наложенного на объем класса выделенных значений. Для экономии места опускаю обобщение этого материала для следования с множественными заключениями и адресую читателя к работам Р. Вуйцицкого [58, § 4.7], а также Д. Шусмита и Т. Смайли [49, $\S 2.1, \S 2.2, \S \S 13.1-13.3]$.

На основе определенного выше понятия следования, О. Ариэли и соавторы вводят понятия пред-отрищания и слабого отрищания [2]. Пусть $\langle\mathcal{L}, \vdash\rangle$ пропозициональная логика, язык $\mathcal{L}$ которой содержит унарную связку ᄀ

- Говорим, что ᄀ есть пред-отрицание, если $p \nvdash \neg p$ для $p \in$ $\operatorname{Var}(\mathcal{L})$.

- Пред-отрицание является слабым отрицанием, если $\neg p \nvdash p$ для $p \in \operatorname{Var}(\mathcal{L})$.

Если $X \vdash \alpha \Longleftrightarrow\langle X, \alpha\rangle \in C n(M)$ для некоторой матрицы $M=\langle\mathcal{A}, D\rangle$, первое условие означает, что $\neg x \notin D$ для некоторо- 
го $x \in D$, а второе - что $\neg y \in D$ для некоторого $y \notin D$. То есть базовым свойством отрицания, если следовать условиям выше, оказывается то, что операция не сохраняет выделенное значение. А дуальное свойство - не сохранять невыделенное значение - «надстраивается» над ним. Это отражает взгляд на истинностные значения, при котором «истина» оказывается главным значением, а «ложь» играет вторичную, подчиненную роль. Подобный взгляд отражен в обычном определении матричного следования - от посылок к заключению сохраняется именно значение «истина». Однако возможен и подход, при котором «истина» и «ложь» трактуются как равноправные значения. В частности, для этого можно использовать следование с множественными заключениями. Сохраняются не только выделенные значения при переходе от посылок к заключениям, но и невыделенные при переходе в обратную сторону. Тогда условия $\langle\{p\},\{\neg p\}\rangle \notin C n_{M}(M)$ (не сохраняется «истина» слева направо) и $\langle\{\neg p\},\{p\}\rangle \notin C n_{M}(M)$ (не сохраняется «ложь» справа налево) оказываются симметричными ${ }^{3}$. С этой точки зрения представляется обоснованным переформулировать условия Ариэли следующим образом:

- Говорим, что $\neg$ есть пред-отрицание, если $p \nvdash \neg p$ или $\neg p \nvdash p$ для $p \in \operatorname{Var}(\mathcal{L})$.

- Пред-отрицание является слабым отрицанием, если одновременно $p \nvdash \neg p$ и $\neg p \nvdash p$ для $p \in \operatorname{Var}(\mathcal{L})$.

Это приводит нас к тому, что каждая матрица из классов $T L_{1}$ и $T L_{2}$ есть матрица пропозициональной логики с пред-отрицанием. На этом основании можно заключить, что все логики, задаваемые рассматриваемыми матрицами, обладают достаточным количеством полезных логический свойств, чтобы оправдать их дальнейшее изучение.

\footnotetext{
3Здесь можно провести параллель с понятиями «негативного объекта справа» и «негативного объекта слева», которые рассматривает Л. Хамберстоун [23, p. 14].
} 
В Части I данной работы большое внимание уделено роли модификаций матриц классической логики в рамках исследований по паранепротиворечивым логикам. Имеет смысл вернуться к этой теме, рассматривая $T L$-матрицы. Тем более, что все известные автору по литературе $T L$-матрицы получены «недооценкой» истинностных значений, а для паранепротиворечивости необходима их «переоценка». Напомню, что в данной работе паранепротиворечивость толкуется в терминах следования: называем пропозициональную логику $\langle\mathcal{L}, \vdash\rangle$ nаранепротиворечивой (относительно $\neg$ ), если найдутся такие $\alpha, \beta \in \operatorname{For}(\mathcal{L})$, что $\{\alpha, \neg \alpha\} \nvdash \beta$. Из определения $\ddot{\neg} x$ (с. 20) очевидным образом вытекает, что матрицы из $T L_{2}$ задают паранепротиворечивые логики.

Однако существует другой подход к определению критериев паранепротиворечивости. Логика считается паранепротиворечивой, если в ней не сохраняются отдельные законы классической логики, например, закон Дунса Скота: $\alpha \supset(\neg \alpha \supset \beta)$. В общем случае условия $\{\alpha, \neg \alpha\} \nvdash \beta$ и $\nvdash \alpha \supset(\neg \alpha \supset \beta)$ не эквивалентны. В логике парадоксов Приста имеет место первое, но не второе. В логике Клини [30] второе, но не первое. С этой точки зрения матрицы из $T L_{2}$ походят на матрицу Приста $L P$.

УтВЕРЖДЕНИЕ 1. Если $M \in T L_{2}$, то $T\left(C_{2}\right) \subseteq T(M)$. Если матрица $M$, к тому же, является $C$-расширяющей, то $T\left(C_{2}\right)=T(M)$.

Доказательство. Докажем первую часть. Функционально эквивалентным образом переопределим матрицу $C_{2}$, заменив $\supset$ на $\subset$ в множестве ее базовых операций. Пусть $\alpha \notin T(M)$. Тогда найдется оценка $h$ в $M$, такая что $h(\alpha)=0$. Определим отображение $\varphi$ из алгебры $M$ в алгебру $C_{2}: \varphi(0)=\varphi(1)=0, \varphi(2)=\varphi(1)$. Из определений $T L_{2}$, $\leftarrow$ и $C_{2}$, вытекает, что $\varphi$ есть гомоморфизм. Поэтому, если $h(\alpha)=0$, то $h^{\prime}\left(p_{i}\right)=\varphi\left(h\left(p_{i}\right)\right)$ есть оценка в $C_{2}$ и $h^{\prime}(\alpha)=0$. Таким образом, $\alpha \in T(M)$ и $T\left(C_{2}\right) \subseteq T(M)$. Докажем вторую часть. Из того, что $M$ является $C$-расширяющей, напрямую следует $T(M) \subseteq T\left(C_{2}\right)$. Вместе с первой частью настоящего утверждения это дает $T\left(C_{2}\right)=T(M)$. 
В исследованиях по противонепротиворечивым логикам важную роль играет понятие максимальности относительно классической логики, восходящее к работам А.М. Сетте [46] и Н. да Коста [10]. Паранепротиворечивую логику $\mathbf{L}=\left\langle\mathcal{L}, \vdash_{\mathbf{L}}\right\rangle$ называют максимальной относительно классической логики $\mathbf{C L}=\left\langle\mathcal{L}, \vdash_{\mathbf{C L}}\right\rangle$, если она отвечает двум условиям:

- Каждая теорема L есть теорема $\mathbf{C L}$.

- Если $\alpha$ теорема $\mathbf{C L}$, но не теорема $\mathbf{L}$, добавление $\alpha$ к $\mathbf{L}$ в качестве аксиомы, превращает $\mathbf{L}$ в $\mathbf{C L}$.

Большая часть известных в литературе многозначных паранепротиворечивых логик обладает этим свойством. В частности, таковы все логики, задаваемые матрицами из класса $8 K b$ [6, р. 78]. Подробному рассмотрению вопроса о максимальности посвящена работа [35]. В свете доказанного выше утверждения о матрицах из $T L_{2}$ для их анализа понятие максимальности относительно классической логики оказывается неподходящим. В его основе лежит понимание логической системы как класса тавтологий. Но с этой точки зрения ни одна из систем, задаваемых матрицами из $T L_{2}$, просто не является паранепротиворечивой, ведь в каждой из них имеют место и закон Дунса Скота, и закон противоречия $(\neg(\alpha \wedge \neg \alpha))$, отсутствия которого в паранепротиворечивой системе прямо требовал да Коста. Однако мы можем получить содержательные результаты, если обратимся к более обобщенной трактовке максимальности паранепротиворечивых логик. Введем следующие определения [2]:

- Говорим, что логика $\mathbf{L}=\langle\mathcal{L}, \vdash\rangle$ максимально паранепротиворечива в слабом смысле, если каждая логика $\mathbf{L}^{\prime}=\langle\mathcal{L}, \Vdash\rangle$, где $\vdash \subseteq \Vdash$ и множество теорем $\mathbf{L}$ является собственным подмножеством множества теорем $\mathbf{L}^{\prime}$, не является паранепротиворечивой.

- Говорим, что логика $\mathbf{L}=\langle\mathcal{L}, \vdash\rangle$ максимально паранепротиворечива в силъном смысле, если каждая логика $\mathbf{L}^{\prime}=\langle\mathcal{L}, \Vdash\rangle$, где $\vdash \subset \vdash$, не является паранепротиворечивой. 
В работе [3] доказано, что каждая матрица из $8 K b$ является максимально паранепротиворечивой в сильном смысле. Ниже я покажу, что это так и для $T L_{2}$.

УтвеРЖДЕНИЕ 2. Если $M \in T L_{2}$, то логика, которую она задает, является максимально паранепротиворечивой в сильном смысле.

ДокАЗАТЕЛЬСТво. Чтобы доказать утверждение, покажем, что имеют место следующие факты: (i) $S_{3}^{\square}$ и все ее функциональные расширения задают логику, максимально паранепротиворечивую в сильном смысле. (ii) $S_{3}^{\star}$ и все ее функциональные расширения задают логику, максимально паранепротиворечивую в сильном смысле. (iii) Каждая матрица из $T L_{2}$ является функциональным расширением $S_{3}^{\square}$ или $S_{3}^{\star}$.

Истинность (i) следует из результатов [3, Th. 3.2]. Переходим к доказательству (ii).

Пусть матрица $M$ для языка $\mathcal{L}$ является функциональным расширением $S_{3}^{\star}$. Пусть существует такая логика $\langle\mathcal{L}, \vdash\rangle$, что $C n(M) \subset \vdash$. Тогда найдутся $X$ и $\alpha$, такие что $X \vdash \alpha$ и $\langle X, \alpha\rangle \notin C n(M)$. В этом случае найдется оценка $h_{0}$ в $M$, при которой $h_{0}(X) \subseteq\{1,2\}$ и $h_{0}(\alpha)=0$. Определим подстановку е следующим образом:

$$
e(p)=\left\{\begin{array}{l}
(p \leftarrow p), \text { если } h_{0}(p)=1, \\
\neg(p \leftarrow p), \text { если } h_{0}(p)=2, \\
p_{0}, \text { если } h_{0}(p)=0 .
\end{array}\right.
$$

$\mathrm{B}$ силу структурности $\vdash$ получаем, что $e(X) \vdash e(\alpha)$. В силу определения $e(p)$, если $h\left(p_{0}\right)=0$, то $h(e(X)) \subseteq\{1,2\}$ и $h(e(\alpha))=0$. Отсюда имеем: $\left\langle\alpha, p_{0}\right\rangle \in C n(M)$. Поскольку $C n(M) \subset \vdash$, также выполняется $\alpha \vdash p_{0}$

Для каждой формулы $\beta$ из $e(X)$ имеет место либо случай (1): если $h\left(p_{0}\right) \in\{1,2\}$, то $h(\beta) \in\{1,2\},-$ либо случай $(2): h(\beta)=0$ при $h\left(p_{0}\right)=1$ или $h\left(p_{0}\right)=2$.

Рассмотрим случай (1). Если $\beta \in e(X)$, то $h(\beta) \in\{1,2\}$ при любом $h$. Следовательно, $\left\langle\left\{q_{0}, \neg q_{0}\right\}, \beta\right\rangle \in C n(M)$. Поскольку $C n(M) \subset \vdash$, также выполняется $q_{0}, \neg q_{0} \vdash \beta$. В силу транзитивности 
и монотонности $\vdash, q_{0}, \neg q_{0} \vdash \alpha$. Но так как $\alpha \vdash p_{0}$, из этого вытекает $q_{0}, \neg q_{0} \vdash p_{0}$. Поэтому $\vdash$ не является паранепротиворечивым.

Рассмотрим случай $(2)$. Если $h(\beta)=0$ при $h\left(p_{0}\right)=1$, то $h \beta\left((p \leftarrow p) / p_{0}\right)=0$ для любой оценки $h$. Если $h(\beta)=0$ при $h\left(p_{0}\right)=2$, то $h \beta\left(\neg(p \leftarrow p) / p_{0}\right)=0$ для любой оценки $h$. Обозначим через $\perp$ формулу, которая принимает значение 0 при любой оценке $h$. Определим подстановку $e^{\prime}$ следующим образом:

$$
e^{\prime}(p)=\left\{\begin{array}{l}
(p \leftarrow p), \text { если } h_{0}(p)=1, \\
\neg(p \leftarrow p), \text { если } h_{0}(p)=2, \\
\perp, \text { если } h_{0}(p)=0 .
\end{array}\right.
$$

При любой оценке $h$ в $M$ каждая $\beta \in X$ принимает выделенное значение и $h(\alpha)=0$. Так как $\left\langle\left\{q_{0}, \neg q_{0}\right\}, \beta\right\rangle \in C n(M),\left\langle\alpha, p_{0}\right\rangle \in C n(M)$ и $C n(M) \subset \vdash$, выполняется $q_{0}, \neg q_{0} \vdash \beta$ и $\alpha \vdash p_{0}$. В силу транзитивности и монотонности $\vdash$ имеем: $q_{0}, \neg q_{0} \vdash p_{0}$. Поэтому $\vdash$ не является паранепротиворечивым.

Теперь докажем (iii). Пусть $M=\langle\{0,1,2\}, \wedge, \vee, \leftarrow, \ddot{\neg},\{1,2\}\rangle$ матрица из $T L_{2}$. Определим операции в матрице $M^{\prime}=$ $\left\langle\{0,1,2\}, \wedge^{\prime}, \vee^{\prime}, \leftarrow^{\prime}, \ddot{\neg},\{1,2\}\right\rangle$ следующими тождествами: $x \wedge^{\prime} y=$ $\ddot{\neg} \ddot{\neg}(x \wedge y), x \vee^{\prime} y=\ddot{\neg} \ddot{\neg}(x \vee y), x \leftarrow^{\prime} y=\ddot{\neg} \ddot{\neg}(x \leftarrow y)$. По определению $T L_{2}, M^{\prime}$ есть $S_{3}^{\square}$, если $\ddot{\neg} x$ есть $\neg^{\square} x$, и $M^{\prime}$ есть $S_{3}^{\star}$, если $\ddot{\neg} x$ есть $\neg S x$. Доказательство закончено.

Можно распространить результаты, сформулированные в утверждениях 1 и 2 на матрицы из класса $T L_{1}$, если трактовать задаваемые ими логики как параполные. Однако, как и в случае паранепротиворечивости, существуют разные формулировки критериев параполноты. В работе [32] параполная логика характеризуется так: «логическая система параполна, если она может служить логикой, лежащей в основе теорий, в которых имеются (замкнутые) формулы, такие что эти формулы и их отрицания одновременно ложны. $<\ldots>$ Кроме того, параполные теории не отвечают принципу исключенного третьего, сформулированному в следующей форме: из двух противоречащих пропозиций одна должна быть истинна» (пер. автора). С формальной точки зрения это соображение может трактоваться 
как запрет на сохранение отдельных законов классической логики, например, $\alpha \vee \neg \alpha$ [47], или $(\alpha \supset \neg \alpha) \supset \neg \alpha$ [4], или $(\neg \alpha \supset \alpha) \supset \alpha$ [8], $[29, \S 2.1]$. Возможно также сформулировать это условие в терминах следования, тогда логику $\mathbf{L}$ называют параполной, если для некотоpых $X \subseteq \operatorname{For}(\mathcal{L})$ и $\alpha, \beta \in \operatorname{For}(\mathcal{L})$ верно, что $X, \alpha \vdash_{\mathbf{L}} \beta, X, \neg \alpha \vdash_{\mathbf{L}} \beta$ и $X \nvdash_{\mathbf{L}} \beta$. Однако нас будет интересовать в первую очередь дуальность между классами $T L_{1}$ и $T L_{2}$. С этой точки зрения в качестве критерия лучше всего подходит следующий принцип: называем логику $\langle\mathcal{L}, \vdash\rangle$ nараполной, е.т.е. $\beta \nvdash\{\alpha, \neg \alpha\}$ для некоторых $\alpha, \beta \in \operatorname{For}(\mathcal{L})^{4}$. Ясно, что такая формулировка имеет смысл, только если расширить определение логики, чтобы следование допускало множественные заключения. Это может быть как уже рассмотренное следование типа $X \vdash Y(X, Y \subseteq \operatorname{For}(\mathcal{L}))$, так и следование с сингулярными посылками и множественными заключениями, которое, в матричной форме, определяется следующим образом ${ }^{5}$ :

$$
C n^{*}(M)=\{\langle\alpha, X\rangle \mid \forall h(h(X) \nsubseteq D \Longrightarrow h(\alpha) \notin D)\} .
$$

Кроме того, следуя [5], введем понятие класса контртавтологий:

$$
T^{*}(M)=\{\alpha \mid \forall h(h(\alpha) \notin D)\}
$$

Это позволяет получить дуальный вариант утверждения 1.

УтВеРЖДЕНИЕ 3. Если $M \in T L_{1}$, то $T^{*}\left(C_{2}\right) \subseteq T^{*}(M)$. Если матрица $M$, к тому же, является $C$-расширяющей, то $T^{*}\left(C_{2}\right)=T^{*}(M)$.

Обратим внимание, что свойство $T^{*}\left(C_{2}\right)=T^{*}(M)$ есть матричный вариант свойства $\alpha \vdash_{\mathbf{L}} \perp \Longleftrightarrow \alpha \vdash_{\mathbf{C L}} \perp$, которое имеет место в интуиционистской логике Int и выступает следствием из известной теоремы Гливенко [56], [55]. Само же утверждение теоремы встречается в литературе в двух вариантах: $\vdash_{\text {Int }} \neg \neg \alpha \Longleftrightarrow \vdash_{\text {CL }} \alpha[40$, p. 391] и $\vdash_{\text {Int }} \neg \alpha \Longleftrightarrow \vdash_{\mathbf{C L}} \neg \alpha$ [22], [55]. Покажем, что при обоих формулировках аналог теоремы доказуем для $C$-расширяющих матриц из $T L_{1}$.

\footnotetext{
${ }^{4}$ Подробный анализ этого вопроса см. в [34] и [24].

${ }^{5} \mathrm{O}$ соотношении между $C n, C n^{*}$ и $C n_{M}$ см. [9].
} 
УтвеРЖДЕниЕ 4. Пусть $M \in T L_{1}$ и матрица $M$ является $C$ расширяющей. Тогда верно следующее: (1) $\neg \neg \alpha \in T(M) \Longleftrightarrow \alpha \in$ $T\left(C_{2}\right) ;(2) \neg \alpha \in T(M) \Longleftrightarrow \neg \alpha \in T\left(C_{2}\right)$.

ДокАЗАТЕЛЬСтво. Построим матрицу $M^{\prime}$, заменив в $M$ класс выделенных значений на $D^{\prime}=\{1,2\}$. По построению $T L_{2}$, базовые операции $M^{\prime}$ отвечают условию стандартности Россера-Тюркетта. Следовательно, $C n_{M}\left(M^{\prime}\right)=C n_{M}\left(C_{2}\right)$. Отсюда $T^{*}\left(M^{\prime}\right)=T^{*}\left(C_{2}\right)$. В то же время $T^{*}\left(M^{\prime}\right)=\{\alpha \mid \forall h(h(\alpha)=0)\}$. Так как, в силу утверждения $3, T^{*}(M)=T^{*}\left(C_{2}\right)$, и каждая оценка в $M^{\prime}$ есть оценка в $M$, также верно, что $T^{*}(M)=\{\alpha \mid \forall h(h(\alpha)=0)\}$. Кроме того, если $M$ есть $C$-расширяющая матрица из $T L_{1}$, ее отрицанием является $\neg$.

Докажем $(1, \Longrightarrow)$. Пусть $\neg \neg \alpha \in T(M)$. Тогда $h(\neg \neg \alpha)=2$ для каждой оценки $h$ в $M$. По определению оценки, $\neg^{\diamond}(h(\neg \alpha)=2)$ По определению $\neg, h(\neg \alpha)=0$, то есть $\neg \alpha \in T^{*}(M)$. В силу утверждения $3, \neg \alpha \in T^{*}\left(C_{2}\right)$. Так как $C_{2}$ есть матрица классической логики, $\alpha \in T\left(C_{2}\right)$.

Теперь докажем $(1, \Longleftarrow)$. Пусть $\beta \in T\left(C_{2}\right)$. Так как $C_{2}$ есть матрица классической логики, $\neg \beta \in T^{*}\left(C_{2}\right)$. В силу утверждения 3 , $\neg \beta \in T^{*}\left(C_{2}\right)$. Так как $T^{*}(M)=\{\alpha \mid \forall h(h(\alpha)=0)\}$, верно, что $h(\neg \beta)=0$ для каждой оценки $h$ в $M$. Тогда, по определению $\neg$, $\neg^{\diamond}(h(\neg \alpha)=2)$. По определению оценки, $(h(\neg \neg \alpha)=2)$. Следовательно, $\neg \neg \alpha \in T(M)$.

Наконец, докажем (2). В силу определения $\neg^{\diamond}$, верно, что $\neg^{\diamond} x=$ $\neg^{\diamond} \neg^{\diamond} \neg^{\diamond} x$. Следовательно, $\neg \alpha \in T(M) \Longleftrightarrow \neg \neg \neg \alpha \in T(M)$. Из этого наблюдения и (1) очевидным образом следует, что $\neg \alpha \in T(M) \Longleftrightarrow$ $\neg \alpha \in T\left(C_{2}\right)$.

Поскольку классы $T L_{1}$ и $T L_{2}$ дуальны, также получаем следующее.

УтвеРЖДЕНИЕ 5. Пусть $M \in T L_{2}$ и матрица $M$ является $C$ расширяющей. Тогда верно следующее: (1) $\neg \neg \alpha \in T^{*}(M) \Longleftrightarrow \alpha \in$ $T^{*}\left(C_{2}\right) ;(2) \neg \alpha \in T^{*}(M) \Longleftrightarrow \neg \alpha \in T^{*}\left(C_{2}\right)$.

Стоит отметить, что при дуализации Int в качестве дуального варианта теоремы Гливенко зачастую рассматривается аналог утвер- 
ждения 1: $\vdash_{\mathbf{L}} \alpha \Longleftrightarrow \vdash_{\mathbf{C L}} \alpha$ [5], [55], [56]. Однако в нашем случае это было бы не вполне корректно. Хотя для $C$-расширяющих матриц из $T L_{1}$ выполняются как только что указанное условие, так и дуальные формулировки теоремы в утверждении 5 , в более общем случае это может быть не иметь места. Например, в матрице Приста $L P$ истинно утверждение 1 , однако ложны обе части утверждения 5 , поскольку $T^{*}(L P)=\emptyset$.

Теперь формулируем дуальный вариант утверждения 2. Для этого потребуется следующее определение: будем называть логику $\mathbf{L}=\langle\mathcal{L}, \vdash\rangle$ максимально параполной в сильном смысле, если каждая логика $\mathbf{L}^{\prime}=\langle\mathcal{L}, \Vdash\rangle$, где $\vdash \subset \Vdash$, не является параполной.

УтвеРЖДЕНИЕ 6. Если $M \in T L_{1}$, то логика, которую она задает, является максимально параполной в сильном смысле.

До настоящего момента мы рассматривали общие свойства матриц, входящих в классы $T L_{1}$ и $T L_{2}$ или их $C$-расширяющие подклассы. Теперь проанализируем внутреннюю структуру этих классов. Для этого обратимся к подходу, который применяется в [54], [53] и [28], и упорядочим изучаемые классы по отношению функциональной вложимости.

Для формулировки результатов необходимо определить матрицу $T L_{1}^{\top}=\left\langle\{0,1,2\}, \wedge, \vee, \rightarrow_{\top}, \neg,\{2\}\right\rangle$. Ее базовые операции совпадают с таковыми в $G_{3}$, за исключением $\rightarrow \top$, которая отвечает следующей таблице:

\begin{tabular}{|c|ccc|}
\hline$\rightarrow_{\top}$ & 0 & 1 & 2 \\
\hline 0 & 2 & 2 & 2 \\
1 & 0 & 2 & 2 \\
2 & 0 & 2 & 1 \\
\hline
\end{tabular}

УтвеРждение 7. Пусть $M \in T L_{1}$. Тогда $T L_{1}^{\top}$ есть функциональное расширение $M$. Если матрица $M$, к тому же, $C$-расширяющая, то $G_{3}$ есть функциональное расширение $M$.

ДокАЗАТЕЛЬСТво. По построению $T L_{1}$, каждая функция $f\left(x_{1}, \ldots, x_{n}\right)$, определимая в $M$, отвечает следующему условию: 


$$
\begin{gathered}
f\left(a_{1}, \ldots, a_{i-1}, 1, a_{i+1}, \ldots, a_{n}\right)=0 \Longleftrightarrow \\
f\left(a_{1}, \ldots, a_{i-1}, 2, a_{i+1}, \ldots, a_{n}\right)=0 .
\end{gathered}
$$

То есть $f\left(x_{1}, \ldots, x_{n}\right)$ сохраняет разбиение $\pi=\{\{0\},\{1,2\}\}$. С.В. Яблонский доказал [59], что класс $U$ всех функций, сохраняющих данное разбиение, предполон в $P_{3}$, классе всех функций на $\{0,1,2\}$. Таким образом, класс операций $M$ с необходимостью включается в $U$.

Как показал М.Ф. Раца [41], класс операций $G_{3}$ представляет собой пересечение класса $U$ с классом $T$ всех $C$-расширяющих функций на $\{0,1,2\}$. Таким образом, любой подкласс $U$, содержащий только $C$-расширяющие функции, включается в класс операций $G_{3}$. Это доказывает вторую часть утверждения.

Кроме того, в процитированной работе Раца показано, что класс операций $G_{3}$ предполон в $U$. Отсюда, поскольку операция $\rightarrow \top$ принадлежит классу $U$, но не принадлежит классу $T$, вытекает, что базовые операции $T L_{1}^{\top}$ образуют базис класса $U$. Это доказывает первую часть утверждения.

Из доказательства пункта (iii) утверждения 2, а также дуальности классов $T L_{1}$ и $T L_{2}$ также вытекает следующее.

УтВеРЖДЕНИЕ 8. Пусть $M \in T L_{1}$. Тогда $M$ есть функциональное расширение $B_{3}^{\diamond}$ или $B_{3}^{\star}$. Если матрица $M$, к тому же, $C$-расширяющая, то $M$ не является функциональным расширением $B_{3}^{\star}$.

Утверждения 7 и 8 позволяют заключить, что матрицы класса $T L_{1}$ образуют решетку по отношению функциональной вложимости, в которой супремумом выступает класс матриц, функционально эквивалентных $T L_{1}^{\top}$, а инфинумом - пустое множество. Подкласс $C$-расширяющих матриц представляет собой подрешетку данной решетки, где супермумом выступает класс матриц, функционально эквивалентных $G_{3}$, а инфинумом класс, состоящий из матрицы $B_{3}^{\diamond}$.

Так как классы $T L_{1}$ и $T L_{2}$ дуальны, матрицы из $T L_{2}$ образуют решетку, изоморфную решетке матриц из $T L_{1}$. Для нее выполняются следующие утверждения. 
УтвеРждение 9. Пусть $M \in T L_{2}$. Тогда матрица $T L_{2}^{\top}$, дуальная $T L_{1}^{\top}$, есть функциональное расширение $M$. Если матрица $M$, к тому же, $C$-расширяющая, то $G_{3}^{*}$ есть функциональное расширение $M$.

УтвеРЖДЕниЕ 10. Пусть $M \in T L_{2}$. Тогда $M$ есть функциональное расширение $S_{3}^{\square}$ или $S_{3}^{\star}$. Если матрица $M$, к тому же, $C$-расширяющая, то $M$ не является функциональным расширением $S_{3}^{\star}$.

Матрицы $B_{3}^{\diamond}$ и $S_{3}^{\square}$, которые являются наиболее слабыми с функциональной точки зрения в своих классах, обладают рядом интересных свойств, на которых стоит остановиться отдельно.

Как и $P^{1}$ и $I^{1}, B_{3}^{\diamond}$ и $S_{3}^{\square}$ задают литеральные паралогики. Р. Левин и И. Микенберг рассмотрели семейство из четырех трехзначных матриц, задающих такие паралогики, которое включает в себя $P^{1}$, $I^{1}, P^{2}, I^{2}$ [33]. Если мы требуем от операций $\wedge, \vee, \rightarrow$ стандартности, то $P^{1}, I^{1}$ будут функционально слабейшими С-расширяющими матрицами литеральных паралогик. Но в более общем случае такими матрицами окажутся $S_{3}^{\square}$ и $B_{3}^{\diamond}$. Между операциями $C_{2}$ и $S_{3}^{\square}$ существует взаимно-однозначное соответствие. Поскольку в $C$-расширяющей матрице $M$ каждой операции $C_{2}$ соответствует по меньшей мере одна операция $M$, в $S_{3}^{\square}$ не определима никакая $C$-расширяющая матрица $M$, не являющаяся функционально эквивалентной $S_{3}^{\square}$. Теперь покажем, что $S_{3}^{\square}$ есть матрица литеральной паранепротиворечивой логики. В то время как $\left\langle\left\{p_{1}, \neg p_{1}\right\}, q\right\rangle \notin C n\left(S_{3}^{\square}\right)$, можно доказать следующее утверждение.

УтВеРЖДЕНИЕ 11. Если ни одна из формул, принадлежащих $X \cup Y$, не является пропозициональной переменной, то

$$
\langle X, Y\rangle \in C n_{M}\left(S_{3}^{\square}\right) \Longleftrightarrow\langle X, Y\rangle \in C n_{M}\left(C_{2}\right) .
$$

ДокАЗАТЕльСтво. Заменив в $S_{3}^{\square}$ класс выделенных значений $D_{S}=$ $\{1,2\}$ на $D_{B}=\{2\}$, получаем матрицу $B_{3}^{\square}$. Имеет место следующее: $h$ есть оценка формулы $\beta$ в $B_{3}^{\square}$, е.т.е. $h$ есть оценка формулы $\beta$ в $S_{3}^{\square}$. Пусть $\beta^{\prime}$ не является пропозициональной переменной. Тогда для каждой оценки $h$ в $S_{3}^{\square}$ и $B_{3}^{\square}$ верно, что $h\left(\beta^{\prime}\right) \in\{0,2\}$. 
Как следствие, $h\left(\beta^{\prime}\right) \in D_{S} \Longleftrightarrow h\left(\beta^{\prime}\right) \in D_{B}$. То есть если ни одна из формул, принадлежащих $X \cup Y$ не является пропозициональной переменной, то $\langle X, Y\rangle \in C n_{M}\left(S_{3}^{\square}\right) \Longleftrightarrow\langle X, Y\rangle \in C n_{M}\left(B_{3}^{\square}\right)$. В то же время $C n_{M}\left(B_{3}^{\square}\right)=C n_{M}\left(C_{2}\right)$.

Итак, $S_{3}^{\square}$ является наиболее слабой с функциональной точки зрения $C$-расширяющей матрицей литеральной паранепротиворечивой логики. В силу дуальности, $B_{3}^{\diamond}$ является слабейшей $C$ расширяющей матрицей литеральной параполной логики.

Теперь вспомним, что матрицы $P^{1}$ и $I^{1}$ можно задать следующим образом: $P^{1}=\left\langle\{0,1,2\}, \supset^{\diamond}, \neg^{\square},\{1,2\}\right\rangle ; I^{1}=\left\langle\{0,1,2\}, \supset^{\square}\right.$, $\left.\neg^{\diamond},\{2\}\right\rangle$, где $\left(x \supset^{\square} y=\neg^{\diamond}\left(\neg^{\diamond} x \subset^{\square} \neg^{\diamond} y\right)\right)$. При этом $P^{1}$ и $I^{1}$ функционально эквивалентны. То есть как обратил внимание А.С. Карпенко [26], $P^{1}$ получается добавлением к $S_{3}^{\square}$ операций из $B_{3}^{\diamond}$, а $I^{1}-$ из $B_{3}^{\diamond}$ при добавлении операций из $S_{3}^{\square}$. На этом основании им предложен метод построения литеральных паралогик с помощью «комбинирования изоморфов классической логики», где изоморфом называется многозначная матрица, порождающая классический класс тавтологий. Дальнейшей разработке этого метода посвящена часть совместной работы Карпенко и Томовой [29, § 3.1.1].

Анализ $T L$-матриц, предложенный в настоящей работе, позволяет внести уточнения в процедуру «комбинирования изоморфов». В случае $P^{1}$, где $D=\{1,2\}$, происходит объединение порождающей классическое отношения следования матрицы $S_{3}^{\diamond}$, которая функционально эквивалентна $B_{3}^{\diamond}$, однако имеет два выделенных значения вместо одного, с паранепротиворечивой $T L$-матрицей $S_{3}^{\square}$. В случае $I^{1}$, где $D=\{2\}$, объединяются трехзначная матрица классической логики $B_{3}^{\square}$, отличная от $S_{3}^{\square}$ лишь тем, что ее класс выделенных значений содержит единственное значение вместо двух, с параполной $T L$-матрицей $B_{3}^{\diamond}$. Паранепротиворечивость и параполнота матриц, полученных «комбинированием изоморфов», является следствием того, что они являются функциональными расширениями соответствующих $T L$-матриц.

Заметим, что функциональными расширениями $T L$-матриц оказываются также все матрицы из рассмотренных в Части I семейств 
$8 K b$ и $8 K b^{*}$. Это вытекает из того, что каждая матрица из $8 K b$ есть функциональное расширение $P^{1}$, а каждая матрица из $8 K b^{*}$ есть функциональное расширение $I^{1}$. Данный факт говорит о том, что $T L$-матрицы могут играть полезную роль в построении функциональных классификаций многозначных логик. Одна такая классификация уже существует в литературе. Это решетка так называемых «естественных $p$-логик», построенная Н. Томовой [52], в которой инфинумом выступает матрица $P^{1}$, и все элементы, таким образом, суть функциональные расширения $T L$-матрицы $S_{3}^{\square}$. Другая решетка логик, принадлежащая тому же автору (см. [53], [54], [13, Гл. 3]), имеет своим инфинумом операции слабой логики Клини, или, что то же самое, внутренние операции логики Бочвара. Матрица с такими операциями и $D=\{1,2\}$ задает класс тавтологий, совпадающий с классическим. При $D=\{2\}$ такая матрица порождает классический класс контр-тавтологий. То есть, хотя эти матрицы и не входят в $T L_{1}$ или $T L_{2}$, они делят существенные свойства с элементами этих классов.

В заключение раздела обратимся к теме, объединяющей Часть I и Часть II этой работы. Хотя ни одна из $T L$-матриц не входит в классы $8 K b$ или $8 K b^{*}$, некоторые из них также могут быть представлены как расширения матрицы классической логики. Это становится возможно благодаря тому, что мы включили в $T L_{1}$ и $T L_{2}$ матрицы, которые не являются $C$-расширяющими. Как следует из доказательства утверждения 7 , в матрице $T L_{1}^{\top}$ выразимы все функции из класса $U$. Множеству этих функций, в частности, принадлежат задаваемые следующими таблицами:

\begin{tabular}{|l|lll|}
\hline$\check{\Lambda}$ & 0 & 1 & 2 \\
\hline 0 & 1 & 1 & 1 \\
1 & 1 & 1 & 1 \\
2 & 1 & 1 & 2 \\
\hline
\end{tabular}

\begin{tabular}{|c|ccc|}
\hline$\check{V}$ & 0 & 1 & 2 \\
\hline 0 & 1 & 1 & 2 \\
1 & 1 & 1 & 2 \\
2 & 2 & 2 & 2 \\
\hline
\end{tabular}

\begin{tabular}{|l|lll|}
\hline$\check{J}$ & 0 & 1 & 2 \\
\hline 0 & 2 & 2 & 2 \\
1 & 2 & 2 & 2 \\
2 & 1 & 1 & 2 \\
\hline
\end{tabular}

\begin{tabular}{|c|c|}
\hline & দُ \\
\hline 0 & 2 \\
\hline 1 & 2 \\
\hline 2 & \\
\hline
\end{tabular}

Нетрудно убедиться, что эти операции отвечают условиям стандартности Россера-Тюркетта. Таким образом, $T L_{1}^{\top}$ есть функциональное расширение трехзначной матрицы классической логики. Заметим, что в $T L_{1}^{\top}$ выразима также следующая операция: $\check{\sim} 0=2$; 


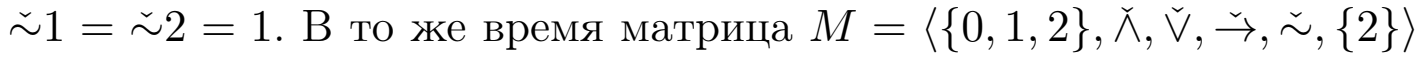
изоморфна матрице $I^{1}$.

В силу дуальности, $T L_{2}^{\top}$ является функциональным расширением трехзначной матрицы классической логики и содержит матрицу, изоморфную $P^{1}$. Последнее означает, что $T L_{2}^{\top}$ представляет собой матрицу логики формальной противоречивости (LFI), которая не входит в семейство $8 K b$.

Итак, мы рассмотрели два класса трехзначных $T L$-матриц для фиксированных пропозициональных языков, а также некоторые свойства логик, задаваемых ими. В заключение рассмотрим направления для обобщения этих результатов. Во-первых, я рассмотрю вопрос о матрицах с большим числом значений. Во-вторых, будут намечены пути перехода от рассмотрения матриц для фиксированного языка к матрицам, где операции трактуются в терминах замкнутых классов функций, без привязки базиса к какой-либо конкретной сигнатуре.

\section{3. Заключение}

Построение аналогов классов $T L_{1}$ и $T L_{2}$ для произвольного $k$ значений и обобщение на них результатов, изложенных в утверждениях 1-11 не представляет заметных затруднений. Обратим лишь внимание на то, что матрица Гёделя $G_{3}$ является супремумом в решетке $C$-расширяющих матриц из $T L_{1}$ (утверждение 7 ), однако уже в четырехзначном случае $G_{4}$ таким свойством не обладает. Дело в том, что операции $G_{4}$ не только сохраняют разбиение $\pi=\{\{0\},\{1,2,3\}\}$ множества $\{0,1,2,3\}$, а также сохраняют его подмножество $\{0,3\}$, т. е. являются $C$-расширяющими, но и сохраняют множество $\{0,2,3\}$. Это значит, что найдется $C$-расширяющая матрица с операциями, сохраняющими $\pi$, которая является собственным функциональным расширением $G_{4}$. Аналогично, требует соответствующей модификации и утверждение 9.

Однако по-настоящему важным следствием увеличения числа истинностных значений будет возможность определить матрицы, которые задают логики, являющиеся паранормалъными, т. е. параполными и паранепротиворечивыми одновременно. В качестве примера 
рассмотрим матрицу, которая содержит $B_{3}^{\diamond}$ и $S_{3}^{\square}$ в качестве подматриц. Возьмем следующие таблицы:

\begin{tabular}{|l|llll|}
\hline$\wedge$ & 0 & 1 & 2 & 3 \\
\hline 0 & 0 & 0 & 0 & 0 \\
1 & 0 & 0 & 0 & 0 \\
2 & 0 & 0 & 3 & 3 \\
3 & 0 & 0 & 3 & 3 \\
\hline
\end{tabular}

\begin{tabular}{|c|cccc|}
\hline$\vee$ & 0 & 1 & 2 & 3 \\
\hline 0 & 0 & 0 & 3 & 3 \\
1 & 0 & 0 & 3 & 3 \\
2 & 3 & 3 & 3 & 3 \\
3 & 3 & 3 & 3 & 3 \\
\hline
\end{tabular}

\begin{tabular}{|c|cccc|}
\hline$\rightarrow$ & 0 & 1 & 2 & 3 \\
\hline 0 & 3 & 3 & 3 & 3 \\
1 & 3 & 3 & 3 & 3 \\
2 & 0 & 0 & 3 & 3 \\
3 & 0 & 0 & 3 & 3 \\
\hline
\end{tabular}

\begin{tabular}{|c|cccc|}
\hline$\leftarrow$ & 0 & 1 & 2 & 3 \\
\hline 0 & 0 & 0 & 3 & 3 \\
1 & 0 & 0 & 3 & 3 \\
2 & 0 & 0 & 0 & 0 \\
3 & 0 & 0 & 0 & 0 \\
\hline
\end{tabular}

\begin{tabular}{|c|c|}
\hline & $\neg x$ \\
\hline 0 & 3 \\
1 & 3 \\
2 & 0 \\
3 & 0 \\
\hline
\end{tabular}

Матрица $M=\langle\{0,1,2,3\}, \wedge, \vee, \rightarrow, \leftarrow, \neg,\{1,3\}\rangle$ задает логику, в которой одновременно имеет место как $\{\alpha, \neg \alpha\} \nvdash \beta$, так и $\beta \nvdash$ $\{\alpha, \neg \alpha\}$. По аналогии с $T L_{1}$ и $T L_{2}$, можно определить целый класс подобных матриц, который мы обозначим $T L_{3}$. На $T L_{3}$ переносится ряд полученных ранее результатов. Матрица $M$ задает литеральную паралогику (аналог утверждения 11). Для каждой $C$-расширяющей матрицы из $T L_{3}$ верно, что ее классы $T(M)$ и $T\left(M^{*}\right)$ совпадают с классическими (аналог утверждений 1 и 3). Каждая матрица из $T L_{3}$ задает максимально паранормальную логику (аналог утверждения 2). Как и в предыдущих классах, в $T L_{3}$ имеются «вырожденные» матрицы. Аналогом $S_{3}^{\star}$ будет такая матрица из $T L_{3}$, что каждая из ее операций выполняет условие $f\left(x_{1}, \ldots, x_{n}\right) \in\{1,3\}$. Аналогом $B_{3}^{\star}$, матрицы из $T L_{1}$, где $f\left(x_{1}, \ldots, x_{n}\right) \in\{0,1\}$, и все неэлементарные формулы принимают только невыделенные значения, будет матрица, в которой $f\left(x_{1}, \ldots, x_{n}\right) \in\{0,2\}$. Интересно, что матрица из $T L_{3}$, где $f\left(x_{1}, \ldots, x_{n}\right) \in\{1,2\}$, не является вырожденной. Она изоморфна матрице $M$, которую мы определили выше.

До настоящего момента мы рассматривали матрицы в фиксированных языках. На примере $\rightarrow$ и $\leftarrow$ мы увидели, что выбор языка, для которого мы строим классические матрицы, играет значительно большую роль, чем в двухзначном случае. Например, класс 
$8 K b^{*}$ оказывается изоморфен классу $8 K b$, только если в первом используется $\leftarrow$ как одна из базовых операций. Поэтому, хотя условия стандартности Россера-Тюркетта и удобны, они не подходят для общего описания многозначных матриц классической логики или $T L$-матриц. В работе [11] предложен альтернативный подход - матрицы, задающие классическое отношения следования, описываются в терминах замкнутых классов функций:

- Если $k$-значная матрица порождает классическое отношение следования, то все ее операции содержатся в предполном классе $P_{k}$, сохраняющем двухчастное разбиение множества-носителя.

- Если матрица $M$ порождает классическое отношение следования, то класс ее операций не содержится ни в одном из прообразов предполных классов $P_{2}$ относительно матричного гомоморфизма из $M$ на двузначную Булеву матрицу.

Это дает нам необходимые и достаточные условия, которым должен отвечать класс операций, чтобы на его основе можно было построить многозначную матрицу классической логики или $T L$-матрицу. Но возможно ли сформулировать аналогичные условия, которые позволят определить, является ли некоторая матрица функциональным расширением классической?

Нетрудно сформулировать достаточное условие: матрица является функциональным расширением классической логики, если класс ее базовых операций содержит как подкласс, выполняющий приведенные выше условия, так и по меньшей мере одну операцию, нарушающую первое из них. Однако многие примеры из Части I показывают, что в общем случае это не так. В то время как матрицы из $8 K b$ явным образом строятся как выполняющие достаточное условие, для матриц $P_{3}, E_{3}$ и $B_{3}$ нам пришлось доказывать наличие формулировок, функционально эквивалентных исходным, демонстрируя выразимость тех или иных операций.

Хотя Я. Калицкий предложил алгоритм, который позволяет построить все функции заданной местности, выразимые посредством некоторого набора функций [25], как показал Н.Р. Емельянов [16], 
в $k$-значной логике при $k>2$ задача о выразимости функции через функции определенной системы является $N P$ трудной задачей. Следовательно, такой же трудностью обладает и задача о соответствии класса операций матрицы достаточным условиям, приведенным выше. Вопрос о более простом критерии, которому должны соответствовать операции матрицы, чтобы она являлась функциональным расширением классической, остается открытым.

\section{Литература}

[1] Девяткин Л.Ю. О конечнозначных логических матрицах, порождающих классическое отношение следования // Логико-философские штудии. 2016. Т. 13. № 2. URL: http://ojs.philosophy.spbu.ru/index.php/lphs/article/view/438 (дата обращения: 15.10.2016).

[2] Девяткин Л.Ю., Карпенко А.С., Попов В.М. Трехзначные характеристические матрицы классической пропозициональной логики // Труды научно-исследовательского семинара Логического центра Института философии РАН. 2007. T. XVIII. С. 50-62.

[3] Девяткин Л.Ю., Преловский Н.Н., Томова Н.Е. В границах трехзначности. М.: ИФ РАН, 2015. 136 с.

[4] Емельянов H.P. О сложности задачи выразимости в многозначных логиках // Доклады Академии Наук СССР. 1985. Т. 282. № 3. С. 525529.

[5] Карпенко А.С. Развитие многозначной логики. М.: ЛКИ, 2010. 448 с.

[6] Карпенко А.С., Томова Н.Е. Трехзначная логика Бочвара и литеральные паралогики. М.: ИФ РАН, 2016. 110 с.

[7] Раца М.Ф. О классе функций трехзначной логики, соответствующем первой матрице Яськовского // Проблемы кибернетики. 1969. Вып. 21. C. $185-214$.

[8] Томова Н.E. О четырехзначных регулярных логиках // Логические исследования. М.: Наука, 2009. Вып. 15. С. 223-228.

[9] Томова Н.Е. Естественные $p$-логики // Логические исследования. Вып. 17. Изд-во ЦГИ, 2011. С. 256-268.

[10] Томова Н.Е. Естественные трехзначные логики: функциональные свойства и отношения. М.: ИФ РАН, 2012. 89 с. 
[11] Яблонский C.В. Функциональные построения в $k$-значной логике // Труды математического института им. В.А. Стеклова. Т. 51. М., 1958. C. 5-142.

[12] Arieli O., Avron A. Three-Valued Paraconsistent Propositional Logics // New Directions in Paraconsistent Logic / Ed. by J.-Y. Béziau et al. Springer India, 2015. P. 91-129.

[13] Arieli O., Avron A., Zamansky A. Maximally Paraconsistent ThreeValued Logics // Proceedings of the Twelfth International Conference on the Principles of Knowledge Representation and Reasoning. Toronto, Ontario, Canada, 2010. P. 310-318.

[14] Arieli O., Avron A., Zamansky A. Maximal and Premaximal Paraconsistency in the Framework of Three-Valued Semantics // Studia Logica. 2011. Vol. 97. No. 1. P. 31-60.

[15] Batens D., De Clercq K., Kurtonina N. Embedding and Interpolation for some Paralogics. The Propositional Case // Reports on Mathematical logic. 1999. Vol 33. P. 29-44.

[16] Brunner A.B., Carnielli W.A. Anti-Intuitionism and Paraconsistency // Journal of Applied Logic. 2005. Vol. 3. No. 1. P. 161-184.

[17] Carnielli W., Coniglio M.E., Marcos J. Logics of Formal Inconsistency // Handbook of Philosophical Logic. Vol. 14. Springer Netherlands, 2007. P. 1-93.

[18] Church A. Non-Normal Truth-Tables for the Propositional Calculus Boletin de la Sociedad Matematica Mexicana. 1953. Vol. 10. P. 41-52.

[19] Ciuciura J. A Weakly-Intuitionistic Logic I1 // Logical Investigations. 2015. Vol. 21. No. 2. P. 53-60.

[20] Cobreros P. Vagueness: Subvaluationism // Philosophy Compass. 2013. Vol. 8. No. 5. P. 472-485.

[21] Da Costa N.C.A. On the Theory of Inconsistent Formal Systems // Notre Dame Journal of Formal Logic. 1974. Vol. 15. No. 4. P. 497-510.

[22] D'Ottaviano I.M.L. The Completeness and Compactness of a ThreeValued First-Order Logic // Revista Colombiana de Matematicas. 1985. Vol. 19. P. 77-94.

[23] D'Ottaviano I.M.L., da Costa N.C.A. Sur un problème de Jaśkowski // Comptes Rendus de l'Académie de Sciences de Paris. Ser. A. 1970. Vol. 270. P. 1349-1353. 
[24] Epstein R.L. The Semantic Foundations of Logic. Vol. 1: Propositional logic. Dordrecht: Kluwer, 1990. 388 p.

[25] Ferguson T.M. Łukasiewicz Negation and Many-Valued Extensions of Constructive Logics // Proceedings of the 44th International Symposium on Multiple-Valued Logic (ISMVL 2014). IEEE Computer Society Press, 2014. P. 121-127.

[26] Finn V.K., Grigolia R. Nonsense Logics and their Algebraic Properties // Theoria. 1993. Vol. 59. No. 1-3. P. 207-273.

[27] Gödel K. On the Intuitionistic Propositional Calculus / Gödel K. Collected works I: Publications 1929-1936 / Ed. by S. Feferman et al. Oxford University Press, 1986. P. 223-225.

[28] Goodman N.D. The Logic of Contradiction // Mathematical Logic Quarterly. 1981. Vol. 27. No. 8-10. P. 119-126.

[29] Goré R. Dual Intuitionistic Logic Revisited // Automated Reasoning with Analytic Tableaux and Related Methods / Ed. by R. Dyckhoff. SpringerVerlag, 2000. P. 252-267.

[30] Humberstone L. The Connectives. MIT Press, 2011. 1512 p.

[31] Hyde D. From Heaps and Gaps to Heaps of Gluts // Mind. 1997. Vol. 106. No. 424. P. 641-660.

[32] Kalicki J. A Test for the Existence of Tautologies According to ManyValued Truth-Tables // Journal of Symbolic Logic. 1950. Vol. 15(3). P. $182-184$.

[33] Karpenko A.S. A Maximal Paraconsistent Logic: the Combination of Two Three-Valued Isomorphs of Classical Propositional Logic // Frontiers of Paraconsistent Logic / Ed. by D. Batens, C. Mortensen, G. Priest, J.-P. van Bendegem. Baldock Research Studies Press, 2000. P. 181-187.

[34] Karpenko A.S., Tomova N.E. Bochvar's Three-Valued Logic and Literal Paralogics: Their Lattice and Functional Equivalence $/ /$ Logic and Logical Philosophy. 2016. URL: http://apcz.pl/czasopisma/index.php/LLP/article/view/LLP.2016.029 (дата обращения: 21.10.2016).

[35] Kleene S.C. On Notation for Ordinal Numbers // The Journal of Symbolic Logic. 1938. Vol. 3. No. 4. P. 150-155.

[36] Kubyshkina E., Zaitsev D.V. Rational Agency From a Truth-Functional Perspective // Logic and Logical Philosophy. 2016. Vol. 25. No. 4. P. 499520. 
[37] Loparic A., da Costa N.C.A. Paraconsistency, Paracompleteness, and Valuations // Logique et Analyse. 1984. Vol. 27. No. 106. P. 119-131.

[38] Lewin R.A., Mikenberg I.F. Literal-Paraconsistent and LiteralParacomplete Matrices // Mathematical Logic Quarterly. 2006. Vol. 52. No. 5. P. 478-493.

[39] Marcos J. Nearly Every Normal Modal Logic is Paranormal // Logique et Analyse. 2005. Vol. 48. No. 189-192. P. 279-300.

[40] Marcos J. On a Problem of da Costa // Essays on the Foundations of Mathematics and Logic 2 / Ed. by G. Sica. Polimetrica, 2005. P. 53-69.

[41] McKinsey J.C.C., Tarski A. On Closed Elements in Closure Algebras // Annals of Mathematics. Second Series. 1946. Vol. 47. No. 1. P. 122-162.

[42] Monteiro A. Sur les Algèbres de Heyting Symétriques // Portugaliae Mathematica. 1980. Vol. 39. No. 1-4. P. 1-237.

[43] Priest G. Logic of Paradox // Journal of Philosophical Logic. 1979. Vol. 8. P. 219-241.

[44] Priest G. Paraconsistent Logic // Handbook of Philosophical Logic / Ed. by Dov M. Gabbay, F. Guenthner. Springer Netherlands, 2002. P. 287393.

[45] Rasiowa H., Sikorski R. The Mathematics of Metamathematics. Warszawa, 1963. 520 p.

[46] Rauszer C. Semi-Boolean Algebras and Their Applications to Intuitionistic Logic with Dual Operations // Fundamenta Mathematicae. 1974. Vol. 83. No. 3. P. 219-249.

[47] Rescher N. Many-Valued Logic. New York: McGraw-Hill, 1969. Reprinted: Aldershot: Gregg Revivals, 1993. 349 p.

[48] Ripley D. Sorting out the Sorites // Paraconsistency: Logic and Applications / Ed. by K. Tanaka, F. Berto, E. Mares, F. Paoli. Springer Netherlands, 2013. P. 329-348.

[49] Segerberg K. A Contribution to Nonsense-Logic // Theoria. 1965. Vol. 31. P. 199-217.

[50] Sette A.M. On propositional calculus $P^{1} / /$ Mathematica Japonica. 1973. Vol. 18. P. 173-180.

[51] Sette A.M., Carnielli W.A. Maximal Weakly-Intuitionistic Logics // Studia Logica. 1995. Vol. 55. P. 181-203.

[52] Shoesmith D.J., Smiley T.J. Deducibility and Many-Valuedness // The Journal of Symbolic Logic. 1971. Vol 36. No. 4. P. 610-622. 
[53] Shoesmith D.J., Smiley T.J. Multiple-Conclusion Logic. Cambrige University Press, 1978. 409 p.

[54] Shramko Y., Wansing H. Entailment Relations and/as Truth Values // Bulletin of the Section of Logic. 2007. Vol. 36. No. 3-4. P. 131-144.

[55] Tomova N.E. A Lattice of Implicative Extensions of Regular Kleene's Logics // Reports on Mathematical Logic. 2012. No. 47. P. 173-182.

[56] Tranchini L. Natural Deduction for Dual-Intuitionistic Logic // Studia Logica. 2012. Vol. 100. No. 3. P. 631-648.

[57] Urbas I. Dual-Intuitionistic Logic // Notre Dame Journal of Formal Logic. 1996. Vol. 37. No. 3. P. 440-451.

[58] Wansing H. Constructive Negation, Implication, and Co-implication // Journal of Applied Non-Classical Logics. 2008. Vol. 18. No. 2-3. P. 341364.

[59] Wójcicki R. Theory of Logical Calculi: Basic Theory of Consequence Operations. Dordrecht: Kluwer, 1988. 474 p. 
L.Yu. DEVYATKIN

\title{
Non-classical Modifications of Many-valued Matrices of the Classical Propositional Logic. Part II
}

\author{
Devyatkin Leonid Yuryevich \\ Department of logic, Institute of Philosophy of Russian Academy of Sciences \\ 12/1 Goncharnaya Str., Moscow, 109240, Russian Federation \\ E-mail: leoniddevyatkin@gmail.com
}

This paper constitutes the second part of the duology dedicated to many-valued matrices of the classical propositional logic regarded as a tool of construction and analysis of non-classical logics. There are many pairs of three-valued matrices which differ only in classes of designated values present in the literature. However, the majority of them induce non-classical consequence relations with respect to either one and two designated values. At the same time, there are matrices of non-classical logics, obtained from matrices of the classical logic by contraction or expansion of the class of designated values. The principal part of the paper is devoted to the two classes of matrices. The first class consists of matrices which would induce the classical consequence given $D=\{1,2\}$, but are regarded as having $D=\{2\}$. The second class is obtained by assuming $D=\{1,2\}$ in matrices inducing the classical consequence for $D=\{2\}$. For the matrices in question I prove the maximality (in the strong sense) of paraconsistency or paracompleteness of logics they define, as well as analogues of Glivenko or Dual-Glivenko theorems. The matrices in classes under consideration form lattices with respect to functional embeddability relation. Some matrices obtained from matrices of the classical logic through modifications of their classes of designated values are shown to have equivalent formulations as functional extensions of matrices of the classical logic.

Keywords: many-valued logics, logical matrices, paraconsistency, paracompleteness 


\section{References}

[1] Arieli, O., Avron, A. "Three-Valued Paraconsistent Propositional Logics", in: New Directions in Paraconsistent Logic, ed. by J.-Y. Béziau et al. Springer India. 2015. pp. 91-129.

[2] Arieli, O., Avron, A., Zamansky, A. "Maximally Paraconsistent ThreeValued Logics", in: Proceedings of the Twelfth International Conference on the Principles of Knowledge Representation and Reasoning, 2010, pp. $310-318$.

[3] Arieli, O., Avron, A., Zamansky, A. "Maximal and Premaximal Paraconsistency in the Framework of Three-Valued Semantics", Studia Logica, 2011, Vol. 97, No. 1, pp. 31-60.

[4] Batens, D., De Clercq, K., Kurtonina, N. "Embedding and Interpolation for Some Paralogics. The Propositional Case", Reports on Mathematical logic, 1999, Vol 33, pp. 29-44.

[5] Brunner, A.B., Carnielli, W.A. "Anti-Intuitionism and Paraconsistency", Journal of Applied Logic, 2005, Vol. 3, No. 1, pp. 161-184.

[6] Carnielli, W., Coniglio, M.E., Marcos, J. "Logics of Formal Inconsistency", in: Handbook of Philosophical Logic, Vol. 14. Springer Netherlands, 2007, pp. $1-93$.

[7] Church, A. "Non-Normal Truth-Tables for the Propositional Calculus", Boletin de la Sociedad Matematica Mexicana, 1953, Vol. 10, pp. 41-52.

[8] Ciuciura, J. "A Weakly-Intuitionistic Logic I1", Logical Investigations, 2015, Vol. 21, No 2, pp. 53-60.

[9] Cobreros, P. "Vagueness: Subvaluationism", Philosophy Compass, 2013, Vol. 8, No. 5, pp. 472-485.

[10] Da Costa, N.C.A. "On the Theory of Inconsistent Formal Systems", Notre Dame Journal of Formal Logic, 1974, Vol. 15, No. 4, pp. 497-510.

[11] Devyatkin, L.Yu. "O konechnoznachnykh logicheskikh matritsakh, porozhdayushchikh klassicheskoe otnoshenie sledovaniya" [On Finite-Valued Matrices Which Induce the Classical Consequence Relation], Logiko-filosofskie shtudii, 2016, Vol. $13 . \quad$ No. 2. [http://ojs.philosophy.spbu.ru/index.php/lphs/article/view/438, accessed on 15.10.2016]. (In Russian)

[12] Devyatkin, L.Yu., Karpenko, A.S., Popov, V.M. "Trekhznachnye kharakteristicheskie matritsy klassicheskoi propozitsional'noi logiki" [Theree-Valued Characteristic Matrices for the Classical Propositional 
Logic], in: Trudy nauchno-issledovatel'skogo seminara Logicheskogo tsentra Instituta filosofii RAN, 2007, Vol. XVIII, pp. 50-62. (In Russian)

[13] Devyatkin, L.Yu., Prelovskii, N.N., Tomova, N.E. $V$ granitsakh trekhznachnosti [Within the Boundaries of Three-Valuedness]. M.: IF RAN, 2015. 136 pp. (In Russian)

[14] D'Ottaviano, I.M.L. "The Completeness and Compactness of a ThreeValued First-Order Logic", Revista colombiana de matematicas, 1985, Vol. 19, pp. 77-94.

[15] D'Ottaviano, I.M.L., da Costa, N.C.A. "Sur un problème de Jaśkowski", in: Comptes Rendus de l'Académie de Sciences de Paris. Ser. A, 1970, Vol. 270, pp. 1349-1353.

[16] Emel'yanov, N.R. "O slozhnosti zadachi vyrazimosti v mnogoznachnykh logikakh" [On the Complexity of the Problem of Expressibility in ManyValued Logics.], Doklady Akademii Nauk SSSR, 1985, Vol. 282, No. 3, pp. 525-529. (In Russian)

[17] Epstein, R.L. The Semantic Foundations of Logic. Vol. 1: Propositional logic. Dordrecht: Kluwer, 1990. 388 pp.

[18] Ferguson, T.M. "Lukasiewicz Negation and Many-Valued Extensions of Constructive Logics", in: Proceedings of the 44th International Symposium on Multiple-Valued Logic (ISMVL 2014). IEEE Computer Society Press, 2014, pp. 121-127.

[19] Finn, V.K., Grigolia, R. "Nonsense Logics and Their Algebraic Properties", Theoria, 1993, Vol. 59, No. 1-3, pp. 207-273.

[20] Gödel, K. "On the Intuitionistic Propositional Calculus", in: Gödel K. Collected works I: Publications 1929-1936, ed. by S. Feferman et al. Oxford University Press, 1986, pp. 223-225.

[21] Goodman, N.D. "The Logic of Contradiction", Mathematical Logic Quarterly, 1981, Vol. 27, No. 8-10, pp. 119-126.

[22] Goré, R. "Dual Intuitionistic Logic Revisited", in: Automated Reasoning with Analytic Tableaux and Related Methods, ed. by R. Dyckhoff. SpringerVerlag, 2000, pp. 252-267.

[23] Humberstone, L. The Connectives. MIT Press, 2011. 1512 pp.

[24] Hyde, D. "From Heaps and Gaps to Heaps of Gluts, Mind, 1997, Vol. 106, No. 424, pp. 641-660. 
[25] Kalicki, J. "A Test for the Existence of Tautologies According to ManyValued Truth-Tables", Journal of Symbolic Logic, 1950, Vol. 15, No. 3, pp. 182-184.

[26] Karpenko, A.S. "A Maximal Paraconsistent Logic: the Combination of Two Three-Valued Isomorphs of Classical Propositional Logic", in: Frontiers of Paraconsistent Logic, ed. by D. Batens, C. Mortensen, G. Priest, J.-P. van Bendegem. Baldock Research Studies Press, 2000, pp. 181-187.

[27] Karpenko, A.S. Razvitie mnogoznachnoi logiki [The Development of Many-Valued Logic]. M.: LKI, 2010. 448 pp. (In Russian)

[28] Karpenko, A.S., Tomova, N.E. "Bochvar's Three-Valued Logic and Literal Paralogics: Their Lattice and Functional Equivalence", in: Logic and Logical Philosophy, 2016. [http://apcz.pl/czasopisma/index.php/LLP/article/view/LLP.2016.029 accessed on 21.10.2016].

[29] Karpenko, A.S., Tomova, N.E. Trekhznachnaya Logika Bochvara $i$ Literal'nye Paralogiki [Three-Valued Logic of Bochvar and Literal Paralogics]. M.: IF RAN, 2016. 110 pp. (In Russian)

[30] Kleene, S.C. "On Notation for Ordinal Numbers", The Journal of Symbolic Logic, 1938, Vol. 3, No. 4, pp. 150-155.

[31] Kubyshkina, E., Zaitsev, D.V. "Rational Agency From a Truth-Functional Perspective", Logic and Logical Philosophy, 2016, Vol. 25, No. 4, pp. 499520.

[32] Loparic, A., da Costa, N.C.A. "Paraconsistency, Paracompleteness, and Valuations", Logique et Analyse, 1984, Vol. 27, No. 106, pp. 119-131.

[33] Lewin, R.A., Mikenberg, I.F. "Literal-Paraconsistent and LiteralParacomplete Matrices", Mathematical Logic Quarterly, 2006, Vol. 52, No. 5, pp. 478-493.

[34] Marcos, J. "Nearly Every Normal Modal Logic is Paranormal", Logique et Analyse, 2005, Vol. 48, No. 189-192, pp. 279-300.

[35] Marcos, J. "On a Problem of da Costa", in: Essays on the Foundations of Mathematics and Logic 2, ed. by G. Sica. Polimetrica, 2005, pp. 53-69.

[36] McKinsey, J.C.C., Tarski, A. "On Closed Elements in Closure Algebras", Annals of Mathematics. Second Series, 1946, Vol. 47, No. 1, pp. 122-162.

[37] Monteiro, A. "Sur les Algèbres de Heyting Symétriques", Portugaliae Mathematica, 1980, Vol. 39, No. 1-4, pp. 1-237. 
[38] Priest, G. "Logic of Paradox", Journal of Philosophical Logic, 1979, Vol. 8. pp. 219-241.

[39] Priest, G. "Paraconsistent Logic", Handbook of Philosophical Logic, Vol. 6. Springer Netherlands, 2002, pp. 287-393.

[40] Rasiowa, H., Sikorski, R. The Mathematics of Metamathematics. Warszawa, 1963. 520 pp.

[41] Ratsa, M.F. "O klasse funktsii trekhznachnoi logiki, sootvetstvuyushchem pervoi matritse Yas'kovskogo" [On the Class of Functions of Three-Valued Logic Corresponding to Jaśkowski's First Matrix], Problemy kibernetiki, 1969, Vol. 21, pp. 185-214. (In Russian)

[42] Rauszer, C. Semi-Boolean Algebras and Their Applications to Intuitionistic Logic with Dual Operations, Fundamenta Mathematicae, 1974, Vol. 83, No. 3, pp. 219-249.

[43] Rescher, N. Many-Valued Logic. New York: McGraw-Hill, 1969. Reprinted: Aldershot: Gregg Revivals, 1993. 349 pp.

[44] Ripley, D. "Sorting out the Sorites", in: Paraconsistency: Logic and Applications, ed. by K. Tanaka, F. Berto, E. Mares, F. Paoli. Springer Netherlands, 2013, pp. 329-348.

[45] Segerberg, K. "A Contribution to Nonsense-Logic", Theoria, 1965, Vol. 31, pp. 199-217.

[46] Sette, A.M. "On propositional calculus $P^{1}$ ", Mathematica Japonica, 1973, Vol. 18, pp. 173-180.

[47] Sette, A.M., Carnielli, W.A. "Maximal Weakly-Intuitionistic Logics", Studia Logica, Vol. 55, 1995, pp. 181-203.

[48] Shoesmith, D.J., Smiley, T.J. "Deducibility and Many-Valuedness", The Journal of Symbolic Logic, 1971, Vol 36, No 4, pp. 610-622.

[49] Shoesmith, D.J., Smiley, T.J. Multiple-Conclusion Logic. Cambrige University Press, 1978. 409 pp.

[50] Shramko, Y., Wansing, H. "Entailment Relations and/as Truth Values", Bulletin of the Section of Logic, 2007, Vol. 36, No. 3-4, pp. 131-144.

[51] Tomova, N.E. "O chetyrekhznachnykh regulyarnykh logikakh" [On FourValued Regular Logics], Logical Investigations, 2009, Vol. 15, pp. 223-228. (In Russian)

[52] Tomova, N.E. "Estestvennye p-logiki" [Natural p-logics], // Logical Investigations, 2011, Vol. 17, pp. 256-268. (In Russian) 
[53] Tomova, N.E. Estestvennye trekhznachnye logiki: funktsional'nye svoistva $i$ otnosheniya [Natural Three-Valued Logics: Functional Properties and Relations], M.: IF RAN, 2012. 89 pp. (In Russian)

[54] Tomova, N.E. "A Lattice of Implicative Extensions of Regular Kleene's Logics", Reports on Mathematical Logic, 2012, No. 47, pp. 173-182.

[55] Tranchini, L. "Natural Deduction for Dual-Intuitionistic Logic", Studia Logica, 2012, Vol. 100, No. 3. pp. 631-648.

[56] Urbas, I. "Dual-Intuitionistic Logic", Notre Dame Journal of Formal Logic, 1996, Vol. 37, No. 3, pp. 440-451.

[57] Wansing, H. "Constructive Negation, Implication, and Co-implication", Journal of Applied Non-Classical Logics, 2008, Vol. 18, No. 2-3, pp. 341364.

[58] Wójcicki, R. Theory of Logical Calculi: Basic Theory of Consequence Operations. Dordrecht: Kluwer, 1988. 474 pp.

[59] Yablonskii, S.V. "Funktsional'nye postroeniya v $k$-znachnoi logike" [Functional Constructions in $k$-Valued Logic], Trudy matematicheskogo instituta im. V.A. Steklova, 1958, Vol. 51, pp. 5-142. (In Russian) 